\title{
Wavepacket basis for time-dependent processes and its application to relaxation in resonant electronic transport
}

\author{
Peter Bokes $a, b *$ \\ a Department of Physics, Faculty of Electrical Engineering and Information Technology, \\ Slovak University of Technology, 81219 Bratislava, Slovak Republic, \\ ${ }^{b}$ European Theoretical Spectroscopical Facility (ETSF, www.etsf.eu)
}

(Dated: December 3, 2018)

\begin{abstract}
Stroboscopic wavepacket basis sets [P. Bokes, F. Corsetti, R. W. Godby, Phys. Rev. Lett. 101, 046402 (2008)] are specifically tailored for a description of time-dependent processes in extended systems like non-periodic geometries of various contacts consisting of solids and molecules. The explanation of the construction of such a basis for two simple finite systems is followed by a review of the general theory for extended systems with continuous spectrum. The latter is further elaborated with the introduction of the interaction representation which takes the full advantage of the time-dynamics built into the basis. The formalism is applied to a semi-analytical example of electronic transport through resonant tunnelling barrier in 1D. Through the time-dependent generalisation of the Landauer formula given in terms of the Fourier expansion of the transmission amplitude we analyze the temporal character of the onset of the steady-state. Various time-scales in this process are shown to be directly related to the energetic structure of the resonant barrier.
\end{abstract}

*E-mail: peter.bokes@stuba.sk 


\section{INTRODUCTION}

Time-dependent density functional theory (TDDFT) allows the formulation of firstprinciples simulations of many-electron problems in the language of non-interacting particles moving in the effective time-dependent Kohn-Sham potential. Actual solution of such a problem requires a particular representation, or a basis set, for one-electron wavefunctions. There are many possibilities to choose from: system independent basis sets like the realspace representation [1] and plane-waves [2, 3], or more-or-less system-specific basis sets like various types of localized orbitals, either of Gaussian type [4] or numerical orbitals [5, 6], ground state canonical Kohn-Sham orbitals corresponding to the studied system, maximally localized Wanier functions [7] and possibly many other. The system independent basis set have the advantage of being general and not preferential to any particular system, which is balanced by the problem that typically one needs very large number of basis functions to obtain converged results. This has been the main motivation in the development of basis sets that are less general, more specialized for the description of the involved physics or chemistry at sufficient precision. With the latter the almost ubiquitous criterion was their description of the ground states properties of the studied system.

In this paper we discuss a new type of basis sets that are specifically tuned for a description of time-dependent processes in extended systems like solids or non-periodic geometries of various contacts consisting of solids and molecules [8]. The major complications for these systems is their size. In the ground state one may hope to use "nearsightedness" of the electrons [9] and study the relevant questions using cluster or periodic models. In nonstationary processes this is not possible in general since electrons may traverse long distances and 'see' arbitrarily remote parts of the system. A problem related to this, and one that is particularly relevant for the description of quantum transport within the TDDFT is the possibility that the exact, or at least sufficiently precise exchange-correlation potential might

need to be very nonlocal [10] even though the numerical importance of this effect in not clear at present [11, 12].

One possible way is to study very large systems and look only at times smaller than the time it takes for the electrons to realise that their box is in fact finite [2, 13, 14]. Another possibility how to treat infinite systems is to employ embedding self-energies [15, 16] that play the role of semi-infinite parts of the total system, often called electrodes, assumed 
to behave in a known way, and the rest, typically a small finite subsystem that can be treated fully within the TDDFT calculation. The stroboscopic wavepacket basis set gives a framework that avoids the use of embedding self-energies in principle and still treat the system as infinite. Individual basis functions are tailored to the time-dependence in the electrodes and the time-propagation of each electron there is analytical. Only in the regions where the system differs from the electrodes it is necessary to propagate the occupied orbitals numerically. Still, even here the wavepacket basis gives a very appealing picture of electrons being redistributed in between different wavepackets due to local disturbances in the effective potential.

In the following section we introduce the concept of stroboscopic construction of a basis set on a very simple examples: two level system consisting of $1 s$ and $2 s$ orbitals of Li atom and a subset of the eigenstates of Harmonic oscillator. In Sec. IIB we review the formalism of the wavepacket basis for any Hamiltonian with a continuum spectrum which is in Sec. IIC further elaborated into interaction representation based on the wavepacket basis. The following two sections give an example of the use of the basis on transport of electrons through a simple resonant potential barrier. We find a simple and direct relation between the Fourier analysis of a transmission amplitude and the temporal behaviour of the relaxation into the current-carrying steady state.

\section{STROBOSCOPIC WAVEPACKET BASIS}

\section{A. Finite systems}

Let us consider a two level system, e.g. the canonical 1s and 2 s orbitals obtained within the ground state DFT calculation of the Lithium atom, $|1 s\rangle$ and $|2 s\rangle$ with eigenenergies $e_{1}$ and $e_{2}$. To obtain the ground state density, we occupy the first orbital twice and the second one once with the result

$$
n(\vec{r})=2|\langle\vec{r} \mid 1 s\rangle|^{2}+|\langle\vec{r} \mid 2 s\rangle|^{2}
$$

In principle, any unitary rotation between these occupied canonical orbitals gives identical density. We will keep one electron, say with its spin down, in the 1s orbital, but the other two, both with spin up, will be placed into new orbitals,

$$
|g\rangle=\frac{1}{\sqrt{2}}(|1 s\rangle+|2 s\rangle)
$$




$$
\left|u^{\prime}\right\rangle=\frac{1}{\sqrt{2}}(|1 s\rangle-|2 s\rangle)
$$

This is, of course, a well known fact. New and interesting point of view arises if we view the state $\left|u^{\prime}\right\rangle$ as the result of time-propagation of the $|g\rangle[29]$

$$
e^{-i \hat{H} \tau}|g\rangle=\frac{1}{\sqrt{2}} e^{-i e_{1} \tau}\left(|1 s\rangle+e^{-i\left(e_{2}-e_{1}\right) \tau}|2 s\rangle\right)=e^{-i e_{1} \tau}\left|u^{\prime}\right\rangle \text { for } \tau=\frac{\pi}{e_{2}-e_{1}} .
$$

This means, we could consider orbitals $|g\rangle$ and $|u\rangle=e^{-i \hat{H} \tau}|g\rangle$ as two orthogonal states which can be filled with electrons. This rather trivial example clearly demonstrates the principal idea of the stroboscopic basis set: to obtain orthogonal basis by means of a propagation from suitably chosen initial state.

The second example where such a construction works for discreet spectra is harmonic oscillator. Consider $N$ consecutive states with eigenenergies $e_{n}=\hbar \omega_{0} n, \quad n=n_{0}, \ldots, n_{0}+$ $N-1$ and eigenstates $\left|\phi_{n}\right\rangle$. We first choose the initial state as the equal combination of the eigenstates,

$$
\left|\psi_{0}\right\rangle=\frac{1}{\sqrt{N}} \sum_{n=n_{0}}^{n_{0}+N-1}\left|\phi_{n}\right\rangle .
$$

In principle, the phases of each of the coefficient in the initial state are arbitrary, which reflects certain freedom in choosing a particular basis.

The remaining members of the new basis are obtained through propagating the initial state with time step $\tau=2 \pi /\left(N \omega_{0}\right)$,

$$
\left|\psi_{m}\right\rangle=e^{-i \hat{H} m \tau}\left|\psi_{0}\right\rangle, \quad m=0,1, \ldots, N-1 .
$$

It is a simple excercise to show that this set is orthogonal, $\left\langle\psi_{m} \mid \psi_{m}^{\prime}\right\rangle=\delta_{m m^{\prime}}$, and spans identical subspace as the chosen eigenstates. The new basis carries certain resemblance with the coherent states used extensively within the quantum optics [17]. Namely, both lead to almost classical interpretation of the dynamics of a single quantum particle, e.g. the contribution of a single electron to the total density is oscillating from right to the left of the parabolic potential with the period $T=2 \pi / \omega_{0}$. On the other hand, in contrast with the coherent states, the time-propagated states by construction consists only from eigenstates with energies within given interval which allows for exact description of non-interacting many-electron ground states or simple non-equilibrium models, as it is described in the following sections. 


\section{B. Infinite systems}

Systems of infinite extent in space have not only discreet but most importantly also continuous spectrum of eigenvalues. In our previous work [8] we have shown that the states with eigenvalues from chosen interval of energies $(\epsilon, \epsilon+\Delta \epsilon)$ in the continuous spectrum can be unitarily transformed into wavepacket basis, in a similar way to the examples in the previous section. In the following we will review the basic steps of this formalism.

Let the system of noninteracting particles under consideration be initially characterised by a reference Hamiltonian $\hat{H}$ with a continuous part of its spectrum covered with intervals of energies $\left(\epsilon_{n}, \epsilon_{n+1}\right), \quad n=0,1, \ldots$ to which we refer here as the energy bands. We will assume that each energy within given band is $N_{n}$ times degenerate. The eigenstates of the reference Hamiltonian,

$$
\hat{H}|\epsilon, \alpha\rangle=\epsilon|\epsilon, \alpha\rangle
$$

need to be taken normalized to the delta-function of their energies,

$$
\left\langle\epsilon^{\prime}, \alpha^{\prime} \mid \epsilon, \alpha\right\rangle=\delta\left(\epsilon-\epsilon^{\prime}\right) \delta_{\alpha, \alpha^{\prime}}
$$

From the above set of eigenstates we can generate the wave-packet basis set (WPB) by first choosing the initial set of wave-packets (WPs)

$$
|n, 0, \alpha\rangle=\frac{1}{\sqrt{\Delta \epsilon_{n}}} \int_{\epsilon_{n}}^{\epsilon_{n+1}} d \epsilon^{\prime}\left|\epsilon^{\prime}, \alpha\right\rangle, \quad n=0,1,2, \ldots
$$

for each energy band $\left\{\left(\epsilon_{n}, \epsilon_{n+1}\right)\right\}_{n}$ and each value of the degeneracy index $\alpha . \Delta \epsilon_{n}=\epsilon_{n+1}-\epsilon_{n}$ represents the width of all the considered energy bands.

The construction of the WPB is completed by forward and backward time propagation of each member of the initial set

$$
|n, m, \alpha\rangle=e^{-i \hat{H} m \tau_{n}}|n, 0, \alpha\rangle, \quad m= \pm 1, \pm 2, \ldots
$$

by regular, band-dependent time steps $\tau_{n}=2 \pi / \Delta \epsilon_{n}$. This choice of time steps guarantees orthonormality of consecutive wave-packets within each band since,

$$
\begin{aligned}
\left\langle n, m, \alpha \mid n, m^{\prime}, \alpha\right\rangle & =\frac{1}{\Delta \epsilon_{n}} \int d \epsilon d \epsilon^{\prime}\left\langle\epsilon, \alpha\left|e^{-i\left(\epsilon^{\prime} m^{\prime}-\epsilon m\right) \tau}\right| \epsilon^{\prime}, \alpha\right\rangle \\
& =\frac{1}{\Delta \epsilon_{n}} \int d \epsilon e^{-i \epsilon\left(m^{\prime}-m\right) \tau}=\delta_{m, m^{\prime}}
\end{aligned}
$$


where we have used the delta-function normalisation (2.7). The same equation also guarantees that WPs with different degeneracy index $\alpha$, as well as WPs from disjunct energy bands are mutually orthogonal. Collecting together all such WPs together with the discreet eigenstates (if they are present) of the reference Hamiltonian we form a complete orthogonal set of states. The regular time steps between individual WPs remind a view through a stroboscope on a continually moving single wavepacket. This analogy led us to refer to such a complete orthogonal set as the stroboscopic wavepacket basis.

A particular example of the stroboscopic basis are the orthogonal wavepackets constructured from the plane waves and used first by Th. Martin and R. Landauer [18]. In fact, this work served as a motivation for generalisation into the stroboscopic construction in the present form.

There are several very attractive properties of this basis that we would like to emphasize: (1) instead of continuous and delta-function normalised states we can work with countable even though infinitely many states that are localized in space and normalised to one; (2) occupying all states from all energy bands below the Fermi energy, $E_{F}$, we recover identical many-particle ground state of non-interacting particles characterised by the reference Hamiltonian; (3) while the total density of such many-particle ground state is time-independent as it should, electron in each WP state in the energy interval $\left(\epsilon_{n}, \epsilon_{n}+\Delta \epsilon_{n}\right)$ can be interpreted as making transition from $m$-th WP to $m+1$-th WP in time $\tau_{n}$. As it will be demonstrated in Section III, all these properties can be very useful for quick and insightful description of non-equilibrium processes in many-electron systems.

\section{Time-dependent wavepacket basis}

In the previous section the wavepacket basis set was constructed in terms of stroboscopic snapshots of time-propagation of initial set of suitably chosen wavepackets. Clearly, this construction is valid for any initial time. This can be exploited even further within the interaction representation where each time-dependent basis function is an evolving wavepacket, and different orthogonal basis functions are time-shifted with respect to each other by $\tau$. This means that any wavefunction $\phi(x, t)=\langle x \mid \phi, t\rangle$ occupied by an electron can be expanded 
as

$$
\langle x \mid \phi, t\rangle=\sum_{i, n, \alpha}\langle x \mid i, \alpha, n ; t\rangle c_{i, \alpha, n}(t)
$$

where $i$ runs over different energy bands, $\alpha$ through different degenerate states within the band and $n$ over different orthogonal wavepackets from the given band $i$. Due to their very definition through time-evolution these time-dependent basis function have the property

$$
\left\langle x \mid i, \alpha, n ; t+\tau_{i}\right\rangle=\langle x \mid i, \alpha, n+1 ; t\rangle
$$

for any real time $t, \tau_{i}$ given by the width of the energy band $i$ and any integer $n$ indexing the various WPs within the band.

There is not much gained from using this particular representation if the actual Hamiltonian of the system is the reference Hamiltonian which generates the wavepacket basis at all times. In such a case the coefficients $c_{i, \alpha, n}(t)$ are either equal 1 , if the considered band $i$ is below the Fermi energy, or equal 0, if the band $i$ is above the Fermi energy. However, when the actual Hamiltonian locally differs from the reference Hamiltonian at times $t>t_{0}$, i.e. the Hamiltonian is

$$
\hat{H}=\hat{H}_{0}+\hat{V}(t)
$$

where $\hat{V}(t) \neq 0$ only in finite part of the overall system, $\Omega \subset \boldsymbol{R}^{3}$, and $t>t_{0}$, the wavefunctions' coefficients will evolve according the Schroedinger equation in the interaction picture

$$
i \frac{\partial}{\partial t} c_{j, \alpha, m}(t)=\sum_{i, \alpha^{\prime}, n}\left\langle j, \alpha, m ; t|\hat{V}(t)| i, \alpha^{\prime}, n ; t\right\rangle c_{i, \alpha^{\prime}, n}(t) .
$$

Since the perturbation $\hat{V}(t)$ is assumed to be nonzero only in $\Omega$, and the wavepackets are localised in space, the above matrix elements are nonzero only for those WPs that have significant amplitude in $\Omega$. The coefficient $c_{i, \alpha, n}(t)$ for times before the corresponding basis function $\langle x \mid i, \alpha, n ; t\rangle$ is evolved by the reference Hamiltonian into the region $\Omega$ will be equal 1 , reflecting the ground state for $t<t_{0}$ imposed by the reference Hamiltonian in the past. During the following time, when the matrix elements of this WP basis function are nonzero, the coefficient $c_{i, \alpha, n}(t)$ will change according to Eq. (2.14). Finally, once the WP basis function $\langle x \mid i, \alpha, n ; t\rangle$ departs from $\Omega$, the coefficient will freeze and will be kept constant once again. 
It is clear from this discussion that the use of the interaction picture representation of the stroboscopic wavepacket basis allows us to translate the time-dependent quantummechanical problem of a system with infinite extent into one that demands numerical solution of finite number of dynamical equations (2.14). We will demonstrate this strategy in the following sections where we address the behaviour of the relaxation into a current carrying steady-state for a model resonant nano-junction at moderate-to-long time-scales.

\section{ELECTRONIC TRANSPORT THROUGH RESONANT TUNNELLING BAR- RIER}

\section{A. Definition of the model}

For the purposes of demonstration of the use of the time-dependent stroboscopic basis we will study a very simple model of resonant tunnelling. In many respects, such a model can be viewed as a prototype of many interesting phenomena observed in the nanoscopic transport through molecules attached to metallic electrodes [19, 20, 21]. The time-dependence in tunnelling has been addressed using various wavepackets by many authors in the past, e.g. [22, 23, 24, 25, 26, 27]. The distinguishing contribution of the present treatment is the correct many-electron occupation corresponding to finite bias and low temperatures and hence its direct consequences for the current-voltage characteristics. The system will be strictly one dimensional, with two delta-function potential barriers localised at $x=0$ and $x=a$ with equal strengths given by a parameter $\lambda$ (see Fig. 1).

Applying voltage to such a model results in decreasing the constant potential to the right of the double barrier by a value $-V$. The value of the potential in between the two potential barriers is chosen to be $-V / 2$. The physics of this choice is that the electrons inside the double barrier will effectively screen the field and the total drop in potential will be symmetrically split into the two barriers where the electronic density is low and hence the screening is weaker.

The simplicity of this model allows for analytical construction of its right- and left- going 
scattering eigenstates for any positive energy $\epsilon$, e.g. for the right-going state we have

$$
\phi_{k}^{R}(x)=\frac{1}{\sqrt{2 \pi k}}\left\{\begin{array}{cc}
e^{i k x}+r(e) e^{-i k x}, k=\sqrt{2 \epsilon} & x<0 \\
C_{1}(\epsilon) e^{i q x}+C_{2}(\epsilon) e^{-i q x}, q=\sqrt{2(\epsilon+V / 2)} & 0<x<a, \\
t(e) e^{i \kappa x}, \kappa=\sqrt{2(\epsilon+V)} & x>a
\end{array}\right.
$$

where all the functions $r(\epsilon), C_{1}(\epsilon), C_{2}(\epsilon)$ and $t(\epsilon)$ can be obtained from the conditions on continuity the wavefunction and finite discontinuity of its derivative at $x=0$ and $a$, and are simple functions of the energy $\epsilon$ of the considered state. Only the transmission amplitude, $t(\epsilon)$, is directly relevant for the present work and its functional form is

$$
t(\epsilon)=\frac{4 k q}{(\kappa+q+2 i \lambda)(k+q+2 i \lambda) e^{i(\kappa-q) a}-(\kappa-q+2 i \lambda)(k-q+2 i \lambda) e^{i(\kappa+q) a}} .
$$

The prefactor of the scattering state $\phi_{k}^{R}(x), 1 / \sqrt{2 \pi k}$, guarantees correct normalisation given by Eq. (2.7). The right- and left- going scattering states at the same energy are examples of two degenerate continuum eigenstates. In the language of the general formulation of the wavepacket basis [Eqs. (2.8), (2.9) ], their indices $R$ and $L$ represent two possibilities of the degeneracy index $\alpha$. The resulting WPs for $\alpha=R$ and $L$ will be arriving to the barrier from the left or right respectively.

The particular values of the parameters will be chosen in the following way: the Fermi energy is set to $E_{F}=1.0$, the strength of the barriers will be varied through values $\lambda=$ 0.1,1.0 and 2.0. We will use two different distances between the barriers, $a_{1}=9.5$ and $a_{2}=10.5$ to clearly demonstrate the different types of relaxation behaviour. Both of these are above the Fermi wavelength $\lambda_{F}=2 \pi /\left(\sqrt{2 E_{F}}\right)=4.44$ to allow for existence of several resonances within the energy interval $\left(0, E_{F}\right)$ at zero bias. In Fig. 2 we show the transmission of such a potential for nonzero bias $V$ and several values of $\lambda$.

\section{B. Non-linear response to abrupt switching-on of the voltage}

In equilibrium, all from the right- and left- moving wavepackets arising from the energy bands below the Fermi energy will be occupied so that overall current is zero. This is not the case in non-equilibrium situation, and nonzero current can be obtained. The calculation of the current alone can be done using the free electron wavepackets (FWP),

$$
\langle x| i, \alpha, n ; t)=\int_{\epsilon_{n}}^{\epsilon_{n+1}} \frac{d \epsilon}{\sqrt{2 \pi k}} e^{i \alpha k x} e^{-i \epsilon t}, \quad k=\sqrt{2 \epsilon}
$$


i.e. the wavepackets where the reference Hamiltonian is that of free electrons $\hat{H}_{0}=$ $-(1 / 2) d^{2} / d x^{2}$. This Hamiltonian characterises the regions to the right and left of the double barrier. (We will use the round bracket to distinguish the FWP from a general WP $|i, \alpha, n ; t\rangle$.) The degeneracy index $\alpha$ for FWPs is equal to + or - and indicates the rightand left- moving FWPs respectively. Within this representation the current due to the energy band or the bias window $\left(E_{F}-V, E_{F}\right)$ (the corresponding time step between neighbouring FWPs is $\tau_{V}=2 \pi / V$ ) is just a simple counting: number of electrons that occupy $n$-th right-going FWP divided by the time it takes for an electron to move from $n$-th to the $n+1$-th FWP,

$$
I_{n}(t)=\frac{N_{n}(t)}{\tau_{V}}=\frac{N_{n}(t)}{2 \pi} V,
$$

where $N_{n}(t)$ is the average occupation of the $n$-th FWP.

Immediately after the switching-on the bias, the evaluation of the current at a chosen position of the FWP and time needs to be analyzed numerically since the time-dependent Hamiltonian will occupy various unoccupied bands of higher energy and these would also contribute to the current. However, it is possible to arrive at a simple expression for the occupations $N_{n}(t)$ for moderate to long time-scales when only WPs in the bias window $\left(E_{F}-V, E_{F}\right)$ contribute to the current. Under these conditions the average occupation is given in terms of the overlaps between the scattering wavepackets corresponding to the final Hamiltonian which includes the barrier and the bias potential, and the FWPs [8],

$$
N_{n}(t)=2 \sum_{m=0}^{-\infty}\left|\left(n,+,\left.i|i, R, m ; t\rangle\right|^{2},\right.\right.
$$

where

$$
\left(i,+, n|i, R, m ; t\rangle=\frac{1}{V} \int_{E_{F}-V}^{E_{F}} d \epsilon t(\epsilon) \exp \left\{-i \epsilon\left(t+(m-n) \tau_{V}\right)\right\}\right.
$$

The $i$-th energy band is understood to be the bias window, the factor 2 stands for spin degeneracy. The Eq. (3.20) is strictly valid only for intermediate and long times times, $t>1 / E_{F}$, as well as only for FWPs that are sufficiently distant from the resonant barriers, $n>a /\left(v_{F} \tau_{V}\right)$. The simplicity of Eqs. (3.19) and (3.20) is striking. The Eq. (3.20) is simply a Fourier analysis of the transmission on the interval of energies that fall within the bias window. Let us introduce the coefficients of this Fourier series as

$$
t_{\eta}(l)=\int \frac{d \epsilon}{V} t(\epsilon) e^{-i \epsilon \eta} \exp \left\{-i \epsilon\left(l \tau_{V}\right)\right\}
$$


where the time is split into the integer multiple of the time step $\tau_{V}$ and the rest, $t=$ $N_{t} \tau_{V}+\eta, \quad 0<\eta<\tau_{V}$. Using this convention we can write the total current at the position of $n$-th FWP as

$$
I_{n}(t)=\sum_{m=0}^{-\infty}\left|t_{\eta}\left(N_{t}+m-n\right)\right|^{2},
$$

which can be readily evaluated. It turns out that this sum converges rapidly: for the here studied resonant barrier the results for the times show in the Fig. 4 and 5 are converged if we take just 15-20 terms. This is an indication that the number of needed WPs to achieve convergence of results does not need to be large. Similar observation has been found in convergence of the ground states density for square potential barrier [28].

We now proceed to discuss the results for two different values of applied voltage. First we choose such a voltage that only one resonance appears in the bias window $\left(E_{F}, E_{F}-V\right)$. Next we increase the voltage and shorten the distance between the two barriers to $a_{2}=9.5$ to capture two resonances. In the long time limit, the current goes into its steady-state value $I_{\infty}$ given by the Landauer formula [8]

$$
I_{\infty}=\frac{1}{\pi} \int_{E_{F}-V}^{E_{F}} d \epsilon|t(\epsilon)|^{2} .
$$

The resulting $I-V$ characteristic for our model is given in the Fig. 3, Whenever new resonance enters the bias window $\left(E_{F}-V, E_{F}\right)$ a rapid increase in the current appears, which is a well known feature in the transport through molecular nano-junctions [20].

The resulting dependence of the current on time, studied at the location of the $n=4$-th FWP $(n=0$ corresponds to FWP located behind the position $x=a$ ) for the bias voltage $V=0.3$ is shown in Fig. 4. In this case only a single resonance enters the bias window, as it is indicated in the inset of the Fig. 4. It takes time $t=4 \tau_{V}$ for the front with nonzero current to appear at the 4-th FWP basis function in the right lead, and the steady-state current is set in with damped oscillations whose period is given by the time step $\tau_{V}$. This is particularly clear for transmissive enough barriers $(\lambda=0.1)$. Such a behaviour is not just an artefact of the WP basis and the choice of the energy band of width $V$; identical voltage-dependent oscillations were found using non-equilibrium Green's function formalism [15]. Increasing the strength of the barriers, i.e. increasing the parameter lambda, the oscillations are suppressed and instead, new time-scale appears in the functional form of the time-dependence: a slow exponential approach towards the steady-state current. The time-constant of this approach 
is given by the width of the resonance present within the bias window $V$. This can be confirmed by inspection of the transmission spectrum (inset of the Fig. 4).

If two resonances are present within the bias window ( $V=0.7$, Fig. 55), the behaviour of the current relaxation is similar to the previous case for highly transmissive barriers. However, increasing $\lambda$ and hence making the barriers more opaque brings in a new timescale: the slow exponential approach to the steady-state value determined by the widths of the resonances is now superposed with oscillations with period slightly larger than $\tau_{V}$. This can be ascribed to the energy difference between the two resonances that are both within the bias window (the inset of the Fig. 55).

The example of electronic transport through resonant barrier shows how the timedependent expression for the current in terms of the Fourier decomposition of the transmission, Eq. (3.21) allows for a very simple analysis of the time-dependent processes involved in the relaxation of the current towards its steady-state value.

\section{CONCLUSIONS}

The stroboscopic wavepacket basis represent a novel basis set specifically tailored for a description of time-dependent processes in extended systems We have explained the idea of the stroboscopic construction on a simple two level system and a finite system consisting of subset of eigenstates of the harmonic oscillator. In the following we have reviewed the general theory for construction of the stroboscopic wavepacket basis for an extended system with continuous spectrum. We have introduced interaction representation within this basis which practically reduces the number of dynamical equations that need to be solved to finite number.

We have applied the time-dependent formalism to semi-analytical example of electronic transport through resonant tunnelling barrier in 1D. The use of stroboscopic basis allows for physically appealing and at the same time mathematically exact formulation of the transport. Furthermore, through the time-dependent generalisation of the Landauer formula in terms of Fourier analysis of the transmission amplitude we could identify the temporal behaviour of the establishment of the steady state with nonzero current where various timescales could be put directly into relation with the energetic structure of the resonant barrier. 


\section{Acknowledgments}

The author wishes to acknowledge fruitful discussions with R. W. Godby, J. Tóbik and P. Dieška. This research has been supported by the NANOQUANTA EU Network of Excellence (NMP4-CT-2004-500198) and the Slovak grant agency VEGA (project No. 1/0452/09). . 
[1] A. Castro, H. Appel, M. Oliveira, C. A. Rozzi, X. Andrade, F. Lorenzen, M. A. L. Marques, E. K. U. Gross, and A. Rubio, Phys. Stat. Sol. B, 2006, 243, 2465-2488.

[2] X. Qian, J. Li, X. Lin, and S. Yip, Phys. Rev. B, 2006, 73, 035408.

[3] Z. Zhou and S.-I. Chu, arXiv:0902.1489v1 [cond-mat.mes-hall], 2009.

[4] M.W.Schmidt, K.K.Baldridge, J.A.Boatz, S.T.Elbert, M.S.Gordon, J.H.Jensen, S.Koseki, N.Matsunaga, K.A.Nguyen, S.Su, T.L.Windus, M.Dupuis, and J.A.Montgomery, J. Comput. Chem., 1993, 14, 1347-1363.

[5] M. Brandbyge, J. L. Mozos, P. Ordejon, and J. T. K. Stokbro, Phys. Rev. B, 2002, 65, 165401.

[6] A. R. Rocha, V. Garcia-Suarez, S. W. Bailey, C. J. Lambert, J. Ferrer, and S. Sanvito, Phys. Rev. B. 73, 085414 (2006), 2006, 73, 085414.

[7] A. Calzolari, N. Marzari, I. Souza, and M. B. Nardelli, Phys. Rev. B, 2004, 69, 035108.

[8] P. Bokes, F. Corsetti, and R. W. Godby, Phys. Rev. Lett., 2008, 101, 046402.

[9] W. Kohn, Phys. Rev. Lett., 1996, 76, 3168-3171.

[10] G. Vignale and M. D. Ventra, Phys. Rev. B, 2009, 79(1), 014201.

[11] N. Sai, M. Zwolak, G. Vignale, and M. D. Ventra, Phys. Rev. Lett., 2005, 94, 186810.

[12] J. Jung, P. Bokes, and R. W. Godby, Phys. Rev. Lett., 2007, 98, 259701.

[13] N. Bushong, N. Sai, and M. D. Ventra, Nano Lett., 2005, 5, 2569.

[14] M. D. Ventra and T. N. Todorov, J. Phys.: Condens. Matter, 2004, 16, 8025-8034.

[15] G. Stefanucci and C. O. Almbladh, Phys. Rev. B, 2004, 69, 195318.

[16] S. Kurth, G. Stefanucci, C.-O. Almbladh, A. Rubio, and E. K. U. Gross, Phys. Rev. B, 2005, 72, 035308 .

[17] R. J. Glauber, Phys. Rev. Lett., 1963, 10, 84-86.

[18] T. Martin and R. Landauer, Phys. Rev. B, 1992, 45, 1742.

[19] M. D. Ventra, S. T. Pantelides, and N. D. Lang, Phys. Rev. Lett., 2000, 84, 979-982.

[20] A. Nitzan and M. A. Ratner, Science, 2003, 300, 1384-1388.

[21] M. Koentopp, K. Burke, and F. Evers, Phys. Rev. B, 2006, 73, 121403(R).

[22] M. Büttiker and R. Landauer, Phys. Rev. Lett., 1982, 49, 1739.

[23] H. Guo, K. Diff, G. Neofotistos, and J. D. Gunton, Appl. Phys. Lett., 1988, 53, 131.

[24] A. P. Jauho and M. Jonson, Superlatt. microst., 1989, 6, 303-307. 
[25] J. A. Stovneng and E. H. Hauge, Phys. Rev. B, 1991, 44, 13582.

[26] R. Landauer and T. Martin, Rev. Mod. Phys., 1994, 66, 217.

[27] G. Garca-Caldern and J. Villavicencio, Phys. Rev. A, 2001, 64, 012107.

[28] See EPAPS Document No. E-PRLTAO-101-013831 at http://www.aip.org/pubservs/epaps.html.

[29] Throughout the paper we use the atomic units a.u., where $m=\hbar=e=1$. 
Fig. 1 Form of the model resonant potential at nonzero bias $V$. The value of the potential inside the barrier is chosen to be halfway between the bias.

Fig. 2 Transmission function for the model resonant potential at zero bias. $E_{F}=1.0$ guarantees that several resonances are occupied in equilibrium, before the bias is switched on.

Fig. 3 The steady-state current-voltage characteristics of the resonant barrier. Rapid increases in current correspond to an emergence of next resonance within the bias window. The green arrows indicate voltages for which we have studied the time-dependent approach to the here indicated values of current.

Fig. 4 Relaxation of the current towards its steady-states value, $I_{\infty}$, for the case when single resonance is present within the bias window. Varying the strength of the barriers alters the character for oscillatory to exponential. The inset shows the transmission spectrum at this value of the bias with the resonance present in the bias window.

Fig. 5 Relaxation of the current towards its steady-states value, $I_{\infty}$, for the case when two resonances are present within the bias window. Varying the strength of the barriers alters the character for oscillatory to exponential with superimposed oscillations that can be ascribed to the energy distance between the two resonances. The inset shows the transmission spectrum at this value of the bias and the two resonances present in the bias window. 


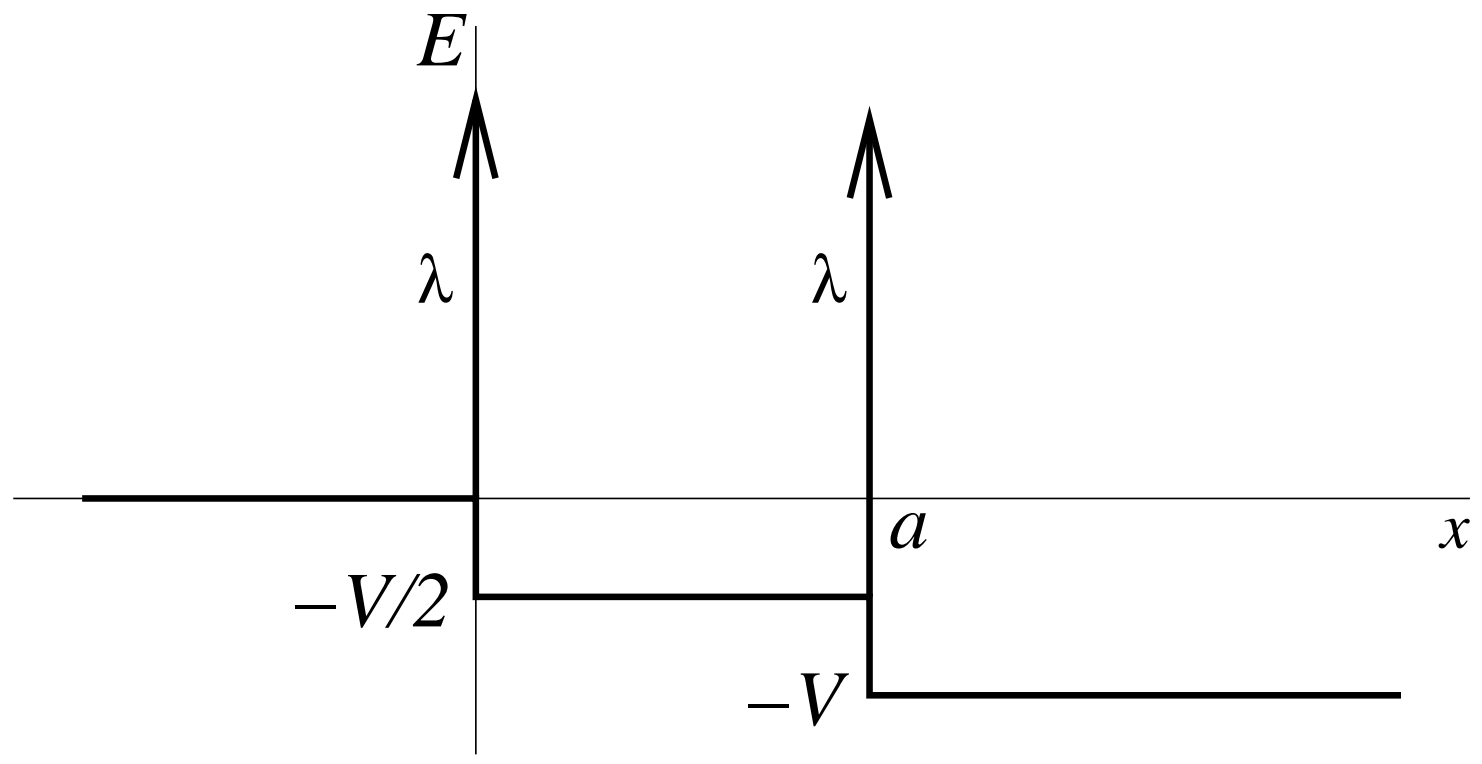

FIG. 1: Form of the model resonant potential at nonzero bias $V$. The value of the potential inside the barrier is chosen to be halfway between the bias.

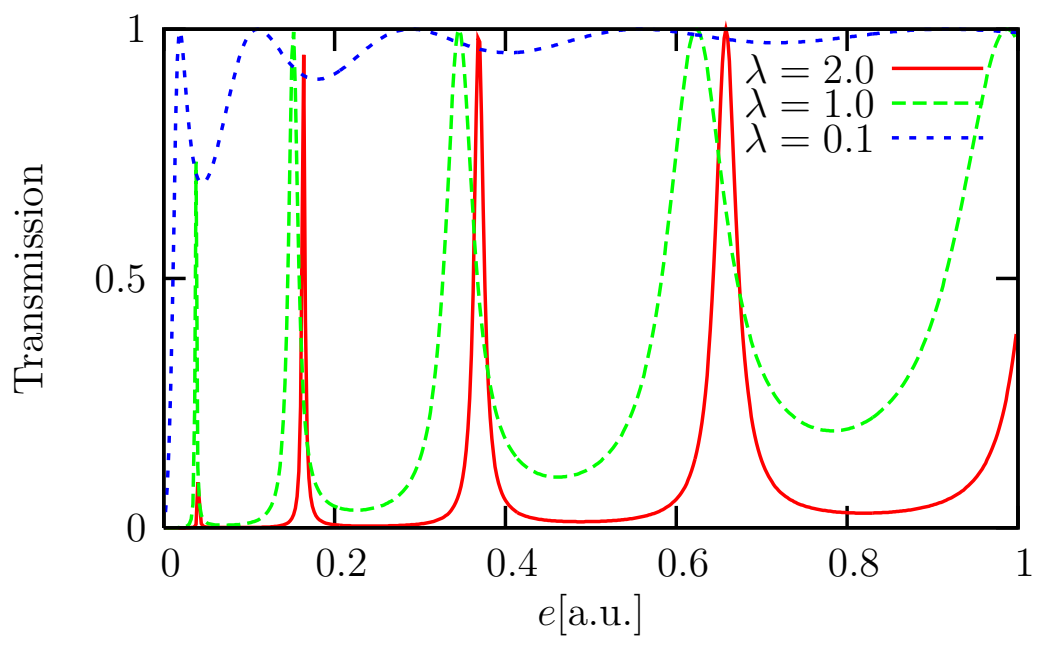

FIG. 2: Transmission function for the model resonant potential at zero bias. $E_{F}=1.0$ guarantees that several resonances are occupied in equilibrium, before the bias is switched on. 


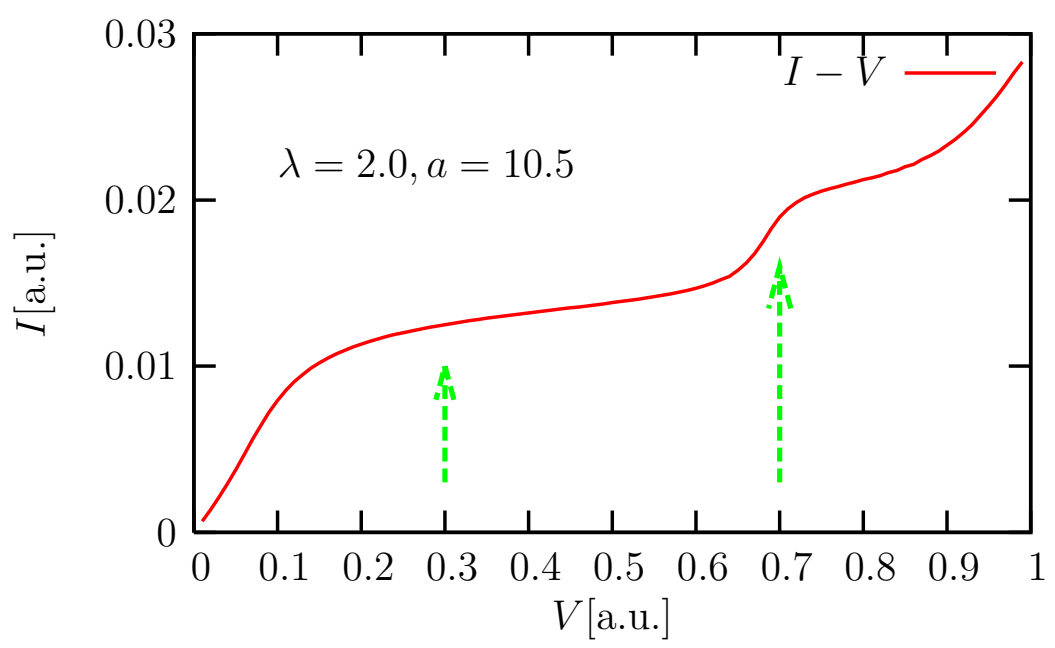

FIG. 3: The steady-state current-voltage characteristics of the resonant barrier. Rapid increases in current correspond to an emergence of next resonance within the bias window. The green arrows indicate voltages for which we have studied the time-dependent approach to the here indicated values of current.

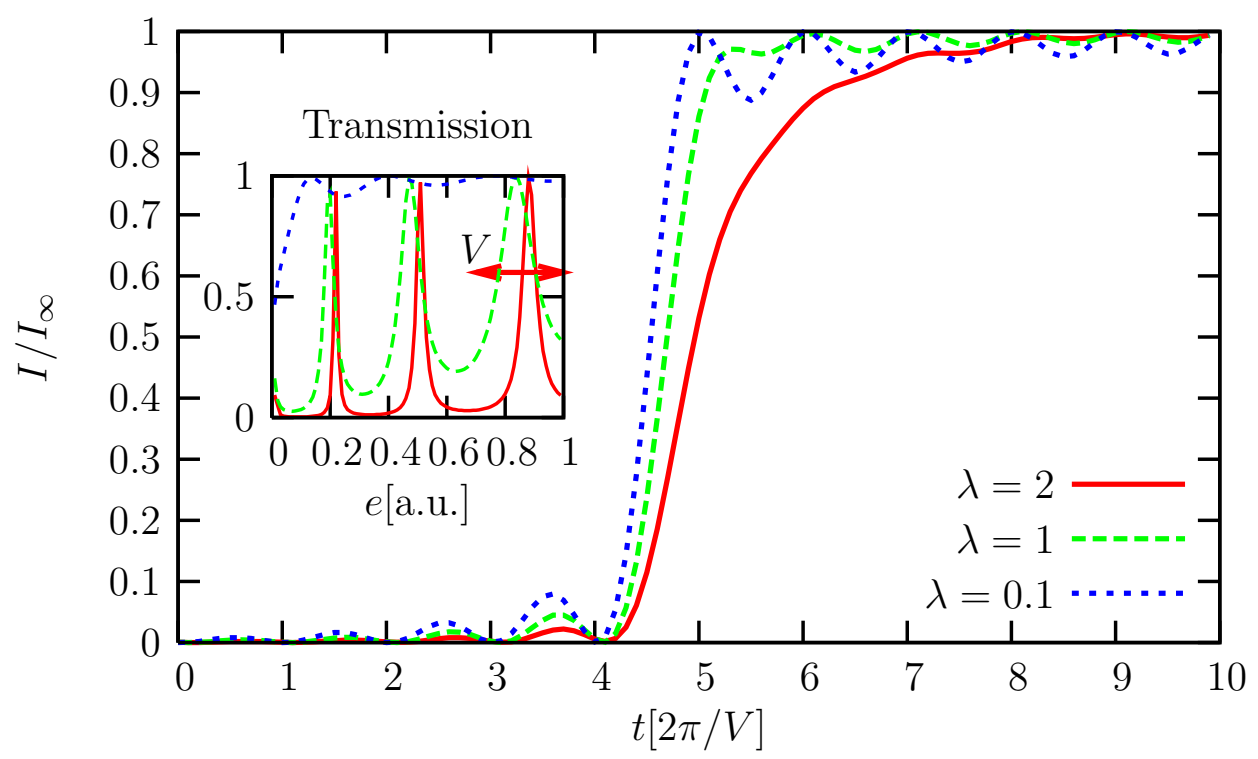

FIG. 4: Relaxation of the current towards its steady-states value, $I_{\infty}$, for the case when single resonance is present within the bias window. Varying the strength of the barriers alters the character for oscillatory to exponential. The inset shows the transmission spectrum at this value of the bias with the resonance present in the bias window. 


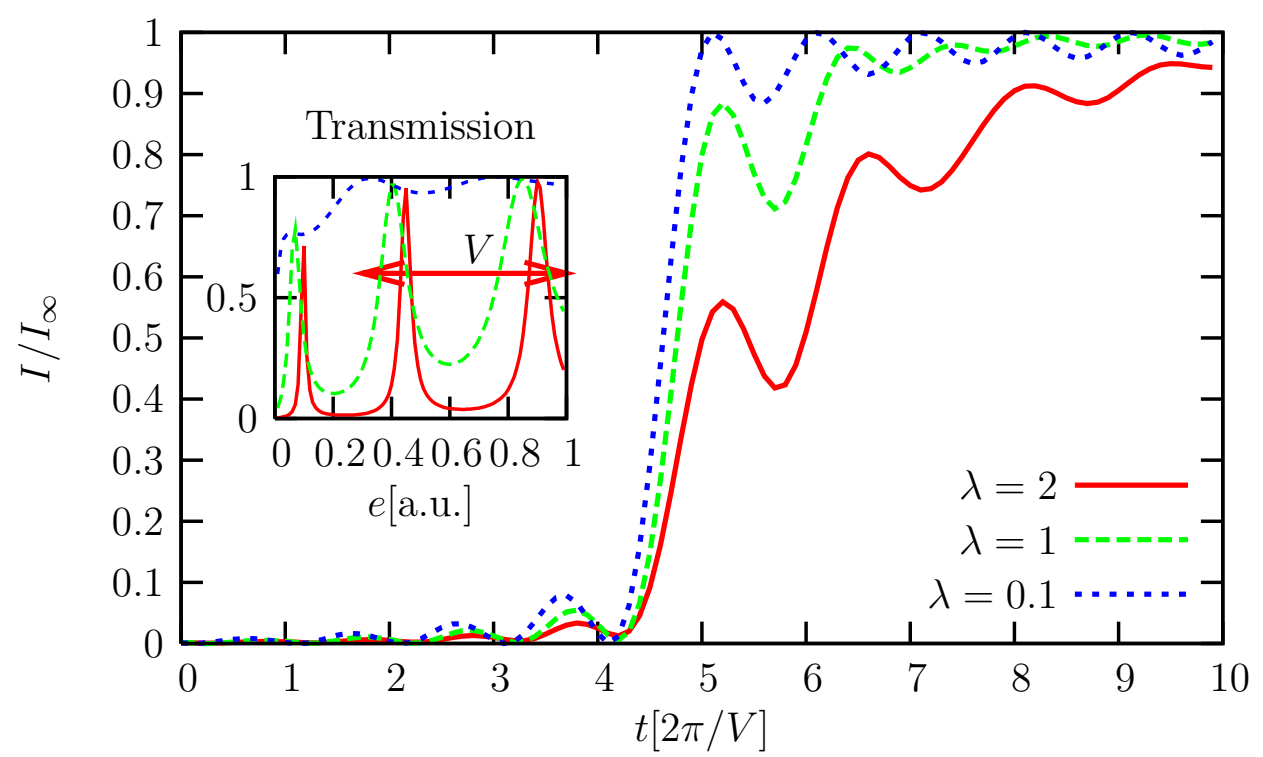

FIG. 5: Relaxation of the current towards its steady-states value, $I_{\infty}$, for the case when two resonances are present within the bias window. Varying the strength of the barriers alters the character for oscillatory to exponential with superimposed oscillations that can be ascribed to the energy distance between the two resonances. The inset shows the transmission spectrum at this value of the bias and the two resonances present in the bias window. 